\title{
A REVIEW OF TWENTY CONSECUTIVE CASES OF ACUTE MASTOIDITIS TREATED BY B.I.P. AND PRIMARY SUTURE.
}

By F. HOLT DIGGLE, F.R.C.S., Aurist Ancoats Hospital, Manchester, late Assistant Surgeon, Ear Hospital, Birmingham, and F. B. GILHESPY, M.R.C.S., L.R.C.P., Assistant Surgeon, Ear and Throat Hospital, Birmingham.

Mr Herbert Tilley, in March 19I9, gave the results of the use of B.I.P. (bismuth, iodoform-paraffin paste) in five cases of acute mastoiditis requiring operation; demonstrating the fact that by its help primary union of the mastoid wound could be obtained even in the most septic cases.

The elimination of the tedious and prolonged period of after dressings which occurred formerly, when the wound was not sewn up at the time of the operation, and the shortened convalescence obtained by the method then described has led to its adoption in many quarters. The object of this communication is to present a series of twenty cases which have been kept under observation for a year or longer after operation, thereby enabling one to form an estimate of the permanent results of the method.

Rationale of Operation.-The cavity in the mastoid bone resulting after the removal of septic material is sterilised with B.I.P., so that the resulting blood clot which fills the cavity shall be sterile. This in turn is replaced by granulation tissue. The wound is treated as sterile and is sewn up at the time of the operation.

Technique of Operation.-It is an advantage to make the incision as near the hair line as possible, as this ensures that the suture line has the support of bone and is not lying over a cavity in the mastoid. The writers have found this incision to be important otherwise there is a tendency to gaping of the wound due to the pressure of the exudation. The mere opening of the mastoid antrum with the escape of pus and the subsequent application of B.I.P. to the cavity is doomed to failure. Anything short of thorough exposure of all infected mastoid cells is certain to result in failure, for, as is well known, the 


\section{F. Holt Diggle and F. B. Gilhespy}

inflammation is not infrequently a generalized osteomyelitis of the whole mastoid process.

The wound is washed out with I in 20 carbolic lotion, and then syringed with methylated spirit and dried. The tip of the index finger is then lightly covered with B.I.P., which is rubbed into the bony cavity and the soft parts.

Subtemporal and subperiostral abscesses are treated in a like manner.

No masses of B.I.P. should be allowed to remain in the wound, and if more than the quantity indicated is used, excessive secretion of serum ensues and cdema, infiltration and redness of the tissues are produced round the wound.

The mastoid incision is then sewn up with interrupted silkworm-gut sutures. These should be inserted closer together than is usual. The external auditory meatus must be disinfected and a paracentesis of the tympanic membrane performed if drainage through the perforation is insufficient.

After Treatment.-The meatus is wiped out once or twice daily with cotton wool, soaked in peroxide of hydrogen. The stitches are removed on the eighth day. The patient can leave the nursing institution on the tenth day, if necessary.

\section{Analysis of Tzenty Cases.}

1. Duration of Stay in Hospital.-The average duration of the stay in hospital of these patients was twelve days. The first two of the series each occupied beds for over twenty days. In these early cases too much B.I.P. was used causing excessive production of serum in the wound, which had to be drained for several days, thereby delaying healing.

Another case remained in hospital for nineteen days, home surroundings were unhealthy, and the child was kept in until a profuse otorrhoa had entirely subsided. Against this a suckling baby of seven months was sent home thirty-six hours after operation and received subsequent attention in the Out-patient Department. The wound healed by primary union.

2. Age of Patients.-This varied from the age of seven months to thirty-five years.

3. Type of Cases.-In all there was a history of acute inflammation of the middle ear, and well-marked symptoms of suppuration in the mastoid antrum were present on admission. In three cases the lateral sinus lay exposed in the 


\section{Acute Mastoiditis treated by B.I.P.}

\begin{tabular}{|c|c|c|c|c|c|c|c|}
\hline 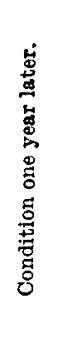 & 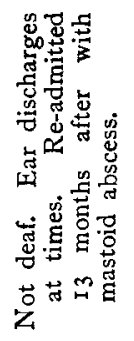 & 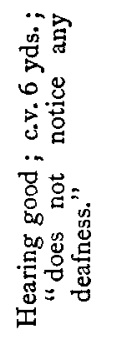 & 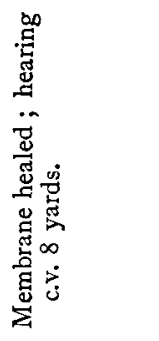 & 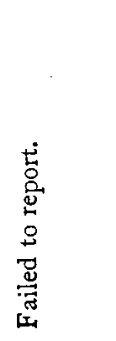 & 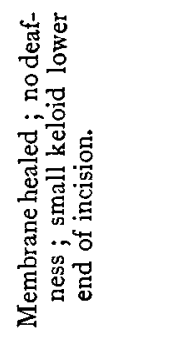 & 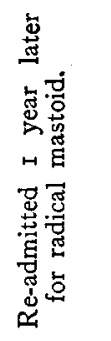 & 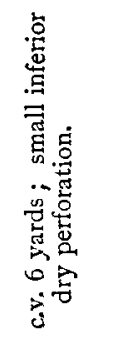 \\
\hline 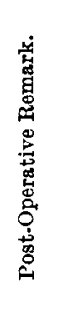 & 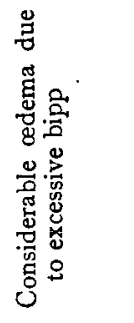 & 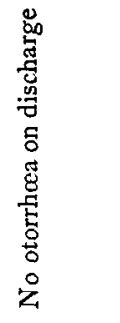 & 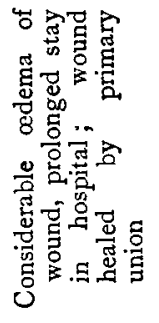 & 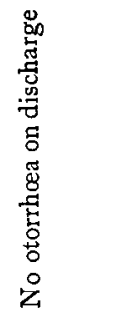 & 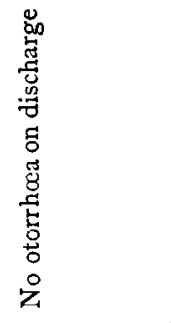 & 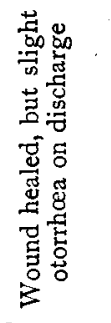 & 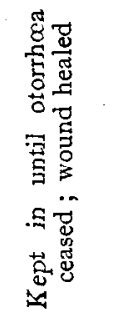 \\
\hline 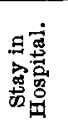 & 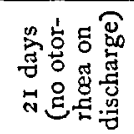 & $\begin{array}{l}\frac{n}{2} \\
\frac{a}{d} \\
\vdots \\
-1\end{array}$ & 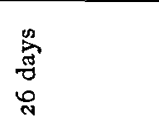 & $\begin{array}{l}0 \\
0 \\
0 \\
0\end{array}$ & 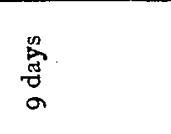 & $\begin{array}{l}\text { 号 } \\
\text { 吾 } \\
\text { 古 }\end{array}$ & 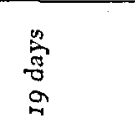 \\
\hline 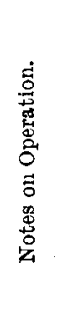 & 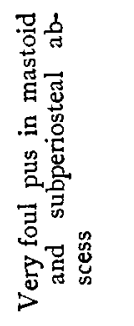 & 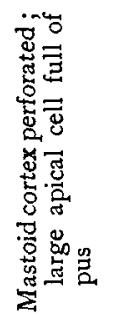 & 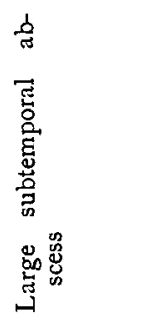 & 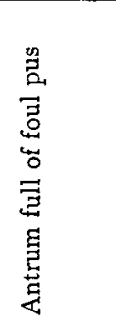 & 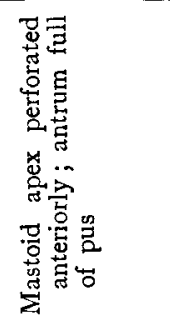 & 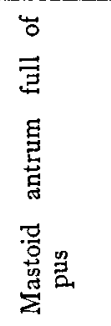 & 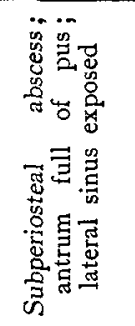 \\
\hline 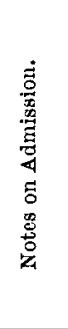 & 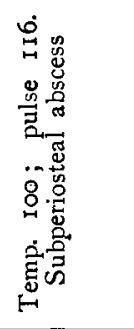 & 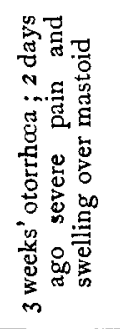 & 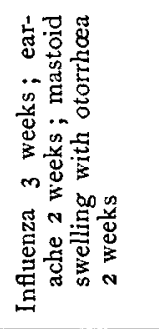 & 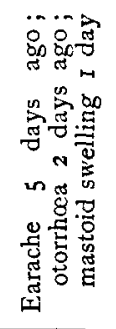 & 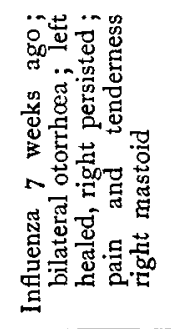 & 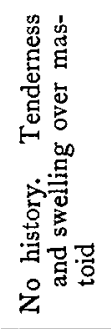 & 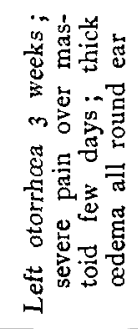 \\
\hline 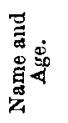 & 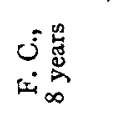 & 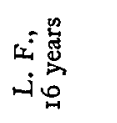 & 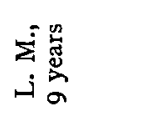 & 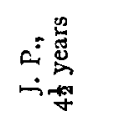 & 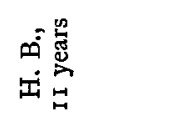 & 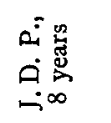 & 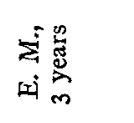 \\
\hline 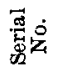 & $\mapsto$ & N & $m$ & $\forall$ & in & 0 & $N$ \\
\hline
\end{tabular}




\section{F. Holt Diggle and F. B. Gilhespy}

\begin{tabular}{|c|c|c|c|c|c|c|c|}
\hline 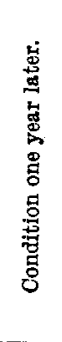 & 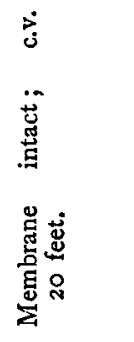 & 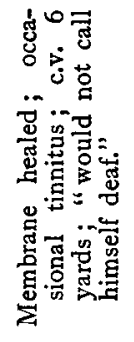 & 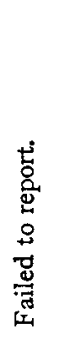 & 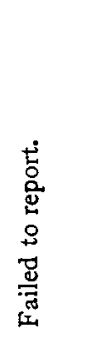 & 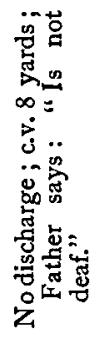 & 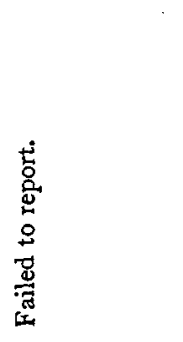 & 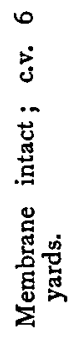 \\
\hline 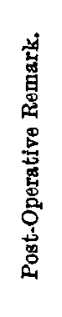 & 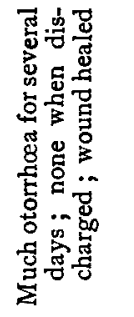 & 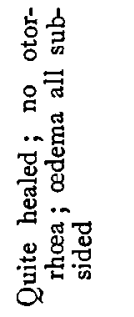 & 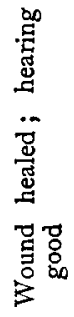 & 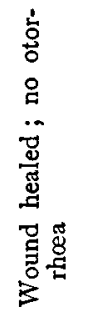 & 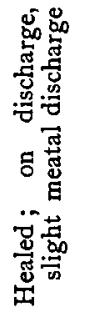 & 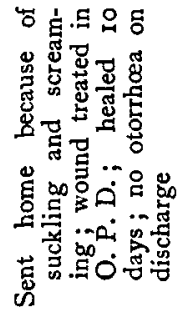 & $\vdots$ \\
\hline 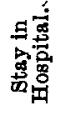 & 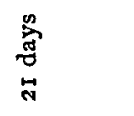 & $\begin{array}{l}n \\
\frac{n}{5} \\
0 \\
0\end{array}$ & 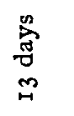 & 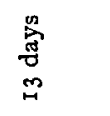 & $\underset{\infty}{\stackrel{0}{J ్ ~}}$ & 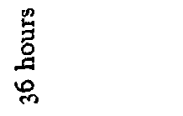 & $\underset{\infty}{\stackrel{\infty}{\vec{J}}}$ \\
\hline 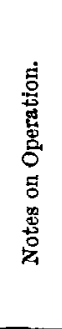 & 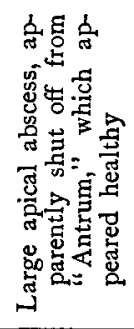 & 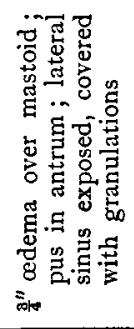 & 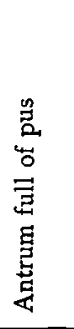 & 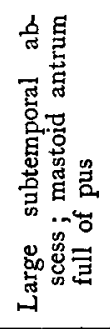 & 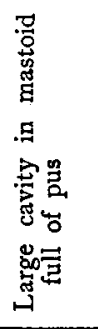 & 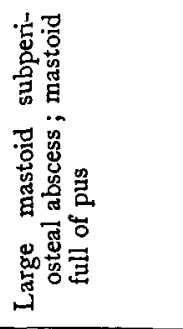 & 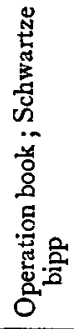 \\
\hline 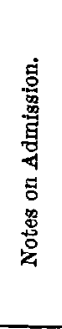 & 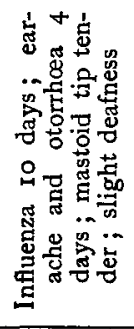 & 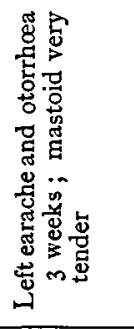 & 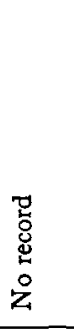 & 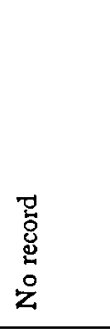 & 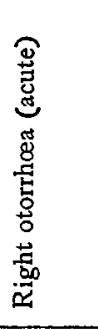 & 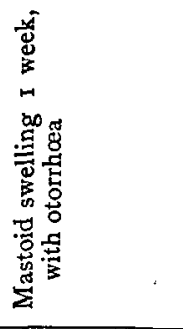 & $\vdots$ \\
\hline 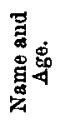 & 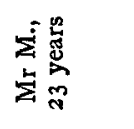 & 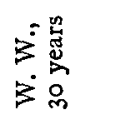 & 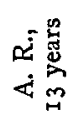 & 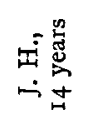 & 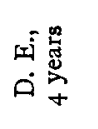 & 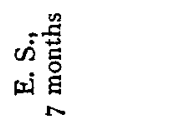 & 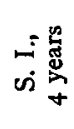 \\
\hline 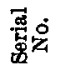 & $\infty$ & $a$ & 요 & 点 & 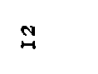 & $\stackrel{m}{m}$ & $\stackrel{H}{ }$ \\
\hline
\end{tabular}




\section{Acute Mastoiditis treated by B.I.P.}

\begin{tabular}{|c|c|c|c|c|c|c|}
\hline 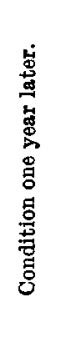 & 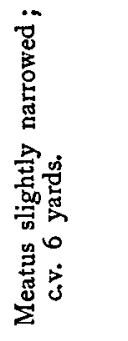 & 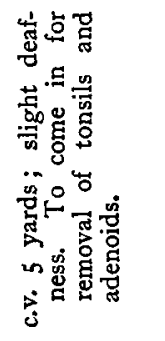 & 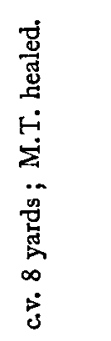 & 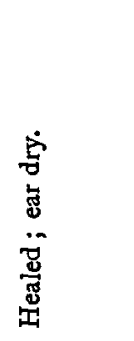 & 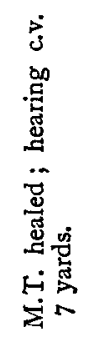 & 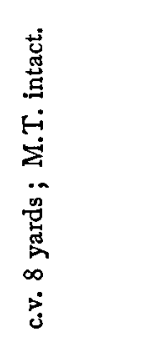 \\
\hline 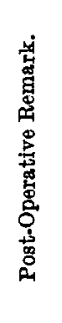 & 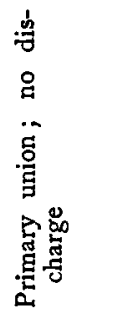 & 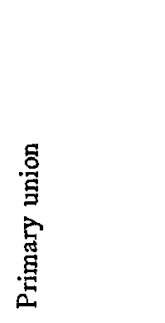 & 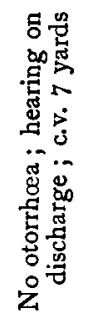 & 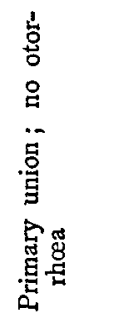 & $\vdots$ & 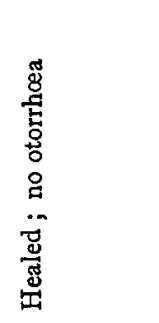 \\
\hline 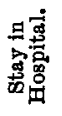 & $\begin{array}{l}\stackrel{n}{5} \\
\stackrel{5}{5} \\
\infty\end{array}$ & 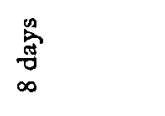 & 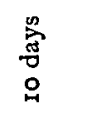 & $\begin{array}{l}\text { 㿿 } \\
\text { 品 }\end{array}$ & $\begin{array}{l}\text { 窝 } \\
\text { 。 } \\
\text { o }\end{array}$ & 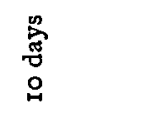 \\
\hline 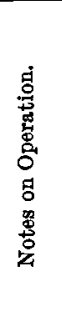 & 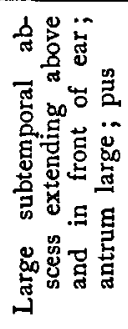 & 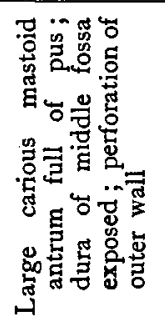 & 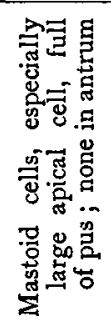 & 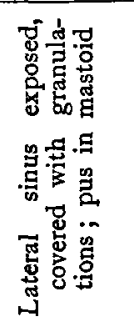 & 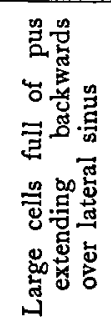 & 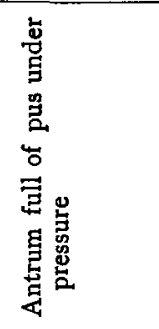 \\
\hline 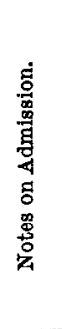 & 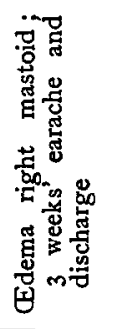 & 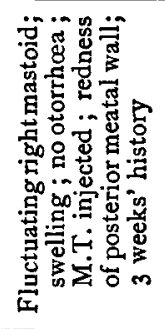 & 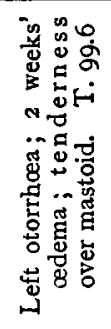 & 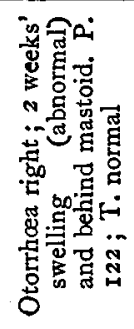 & 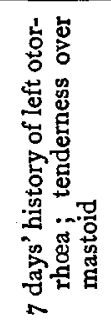 & 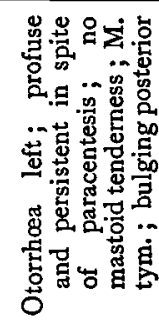 \\
\hline 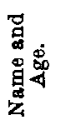 & 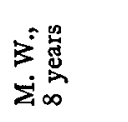 & 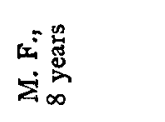 & 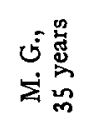 & 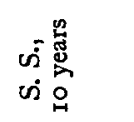 & 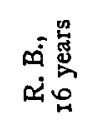 & 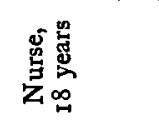 \\
\hline 品安 & $\cong$ & $\stackrel{\circ}{\sim}$ & $\Xi$ & $\stackrel{\infty}{+\infty}$ & o & 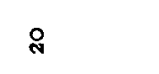 \\
\hline
\end{tabular}




\section{F. Holt Diggle and F. B. Gilhespy}

mastoid cavity and was covered by a layer of granulation tissue; nevertheless no trouble in the sinus followed the closure of the wound; in other words, the septic process had been completely arrested and did not recur after closure of the previously infected cavity. Of course, the method is not applicable in cases of sinus thrombosis. The dura mater was found exposed in two cases. A large subtemporal abscess was present on several occasions.

Edema of the tissues over the mastoid was marked in 50 per cent. of the cases. This went down rapidly after operation.

4. Hearing:-At the discussion; which followed the introduction of this method by $\mathrm{Mr}$ Tilley, it was suggested that B.I.P. might have a bad effect on the hearing. In the present series of cases seen one year after the operation, it is most conclusively seen that the fear was groundless. A few of the statements made by the patients or their parents are given"does not notice any deafness," "is not deaf," "would not call himself deaf." Low conversational voice was heard at six yards on the affected side in all cases.

5. Keloid Formation.-The irritation of B.I.P. has not resulted in the formation of keloid in the line of incision. It may be remarked that the lower the wound is carried in any operation in the mastoid region the more liability is there to keloid formation in the lower end of the incision.

6. Recurrence of Mastoid Trouble. - When brought for inspection thirteen months after operation, F. C., aged 8 years, was brought up with a mastoid swelling. The signs were those of a cold abscess. After opening a large subtemporal abscess, the cavity in the mastoid bone was found lined with pale leathery granulation tissue. The mother said that the child had suffered from a cold for a fortnight previously.

J. D. P., aged 8 years, was seen eleven months after operation, when the mother brought the child to the hospital because of swelling over the mastoid. It was found necessary to perform a radical operation owing to the widespread infection present. In this case the discharge had not cleared up after the initial operation and the surgeon in charge of the case had been given an erroneous history, the discharge from the ears having been more chronic than he was led to believe.

Conclusions.-I. The method was put forward by the originator to demonstrate a means whereby the after treatment 


\section{Acute Mastoiditis treated by B.I.P.}

of cases of "acute mastoid" could be shortened. This advantage has been convincingly demonstrated. In a busy hospital where 200 mastoid operations are performed a year, the saving of beds and labour is enormous. 2. The granulations which fill, or partially fill, the mastoid cavity are not proof against a further invasion of infection by "way" of the Eustachian tube. In every operation on the mastoid antrum the condition of the posterior nares and throat should be attended to. 